\title{
Optimizing Area Power Consumption in LTE type Cells
}

\author{
Tony Keenan and Rudi Villing \\ Callan Institute, Department of Electronic Engineering \\ National University of Ireland Maynooth, (NUIM), Maynooth, Co. Kildare \\ E-mail: tkeenan@eeng.nuim.ie
}

\begin{abstract}
As wireless networks evolve to deal with increasing traffic demand and an increasing number of users, energy efficiency is becoming increasingly important. Area power consumption, defined as the ratio of total radio access network power consumption to the area covered, is a key metric for comparing the energy efficiency of different network designs. This paper examines the optimization of an LTE type cell for energy efficiency by minimizing the area power consumption (APC) subject to user coverage and capacity constraints. First we show that operating the base station at the maximum transmit power (and cell size) which services all users optimizes APC. Next we show that APC is optimum when the base station operates with the minimum bandwidth necessary to service all users. Finally we show that a modified admission scheme (which increases the minimum SINR at which a user will be serviced) improves APC by increasing the maximum capacity and size of cells that can be supported. In addition to improving energy efficiency, the modified scheme requires fewer cells in new or overlay deployments and may potentially permit removal of cells from an existing network.
\end{abstract}

Keywords - Area power consumption, LTE, edge SINR

\section{INTRODUCTION}

Energy efficiency is becoming increasingly important in the design of future wireless networks as the demand for higher capacity wireless services increases. According to [2], 0.12 per cent of global energy demands could be attributed to the mobile telecommunications sector in 2007. Global mobile traffic is expected to continue increasing rapidly resulting in a significant increase in the associated energy demands. For example global mobile data traffic is expected to increase from 0.9 to 11.2 Exabyte's per month by 2017 [3] driven in part by a projected 7 trillion wireless devices worldwide serving 7 billion users [4].

While modern standards such as Long Term Evolution (LTE) have been developed to deliver high capacity services through increased spectral efficiency, the majority of the increased capacity required will have to be achieved through additional wireless infrastructure [5, 6]. However, wireless infrastructure and in particular base stations account for over $70 \%$ of a mobile operators network energy cost [7]. Therefore base stations contribute a significant proportion to the total cost of operating a mobile network. Base stations and mobile networks must be designed efficiently to ensure their significant energy costs are kept low.

As a consequence, the literature shows growing interest in the design and optimization of energy and cost efficient networks. Traditionally the power consumption of a base station or network is used in the optimization of networks. However when designing energy efficient networks and comparing various network designs, in particular heterogeneous networks, a more suitable metric is area power consumption proposed by Richter et al in [8], alternatively called power per area unit [9]. This has become a popular metric and is useful for evaluating different base station designs and identifying energy efficient design trends which can be applied in real world network design. Although the literature using this metric typically considers area coverage planning it has not considered (user and traffic) capacity planning in detail [8, 10-13].

In [8] the impact of the macro base station inter-site distance and the number of micro base stations on the area power consumption of a network was analysed. The relationship between area power consumption and area spectral efficiency was also examined. This work was extended by Richter et all in [12] where the relationship between area power consumption and the traffic dependent spectral efficiency is investigated. While spectral efficiency is used as a means of examining capacity in these works, this metric is not particularly useful if the performance of a set of users with particular traffic demands is to be analysed. In addition these works did not use real world standards in their analysis and aspects such as scheduling issues and control channel 
limitations could not be considered. Arnold et all [13] carried out similar analysis to that in [8] using a more sophisticated base station consumption model but detailed capacity analysis was again not the main focus of the work. The Green Radio Access Network Design Tool presented in [10] can be used to identify the optimum network configuration (the set of base stations and the configuration of each) to provide energy efficient coverage of a given area. In this context, the optimum network configuration is the one with the lowest ratio of total energy consumption to area covered. The tool may be used to compare the optimum network configuration of various different standards given a constraint of a minimum user throughput which must be achieved in any cell. Importantly, however, this does not allow for detailed traffic analysis.

The importance of capacity planning and detailed traffic analysis in the design of networks has long been established in industry and academia. For example, [14, 15] show the importance of both traffic type and resource scheduling on the achievable capacity in a Mobile WiMAX cell. In particular the underlying population of users requiring service and their traffic demands are key factors which must be considered in the design and analysis of any network.

This paper examines the optimization of base station configuration which minimizes area power consumption for a given user traffic demand. Section II introduces the optimization methodology which is based on model based simulation of an LTE type cell with certain simplifications. Thereafter the effect of transmit power and bandwidth allocation on the area power consumption are investigated. Section III tests whether or not a modified admission scheme, first described in [16], can improve the area power consumption (subject to certain constraints being satisfied). Finally, section IV concludes the paper.

\section{OPTIMIZING AREA POWER CONSUMPTION}

There are number of parameters which can be tuned to optimize key performance indicators when designing any base station or network of base stations. Two of the most important parameters in the planning of a base station are its transmit power and bandwidth. This section examines the relationship between transmit power, bandwidth, and the minimum area power consumption that can be achieve for a base station while satisfying specified capacity (user traffic demand) constraints. The methodology is broadly similar to that used in [16] for evaluating spectral efficiency of an LTE type cell but a more sophisticated model has been used.

\section{a) Simulation Model}

The model simulates an LTE type multi cell network of 3-sectored macro base stations. For the purposes of this paper this multi-cell network consists of a central 3-sectored base station surrounded by a number of interfering 3-sectored base stations. The 3 sectored cells of the central base station will be optimized in subsequent sections. Single base station optimization is useful for analysing ideal base station designs and giving insight into the type of design that should be used in either multi base station green field design or existing network modification. The inclusion of multi base station network complexities such as inter-cell interference prevents the model from being over simplified and allows for realistic evaluations. All interfering sectors are assumed to have the same transmit power and a frequency reuse pattern of $(1,3,3)$ is used throughout the network (i.e. each base station is allocated the full bandwidth and this bandwidth is then subdivided and shared equally by the base station's 3 sectors).

All key metrics are calculated for each sector of the central base station while all other base station sectors act as interferers contributing to the inter-cell interference (ICI). In addition to the base station network, the model simulates a user traffic demand which is used for capacity analysis, specifically to determine whether the cell can service all the users in its coverage area or not. In this model, the user traffic demand is characterized by a random uniformly distributed population of users (parameterized by the user density) and a common per user traffic profile (parameterized by the packet size, packet interval, and maximum acceptable latency). It is worth noting that the user traffic demand focuses on users connected to or seeking connection to the network, it does not consider the possibly much larger set of standby users known to the cell but not actively contributing to the traffic demand. 
For a simulation run to be carried out the design parameters outlined in Table 1 must be defined for each base station. Most of these parameters are constant across simulations during the optimization procedure. The notable exceptions are the transmit power and bandwidth (per sector) which are varied during the optimization procedure for the central base station but are held constant for the surrounding interfering base stations.

A simulation run using the model proceeds as follows. First the coverage area of the 3-sectored central base station is calculated. The coverage area, $A_{C}$, is calculated as the total area where the received signal to interference and noise ratio (SINR) is greater than the minimum SINR required by the standard. The SINR is calculated using a link budget analysis and includes ICI due to the interfering base stations.

Based on the user density, a population of users will usually be generated once for a given total area, and all simulations for that area will use this population. All users within the coverage area of a sector are assumed to want service and this set of users is referred to as the active subscriber list. The active subscriber list is found for each sector in the base station.

Next the number of connected users, $C_{\text {users }}$, whose traffic demand can be satisfied is determined for each cell. Unlike the method used in $[16,17]$ the model calculates the theoretical maximum number of connected users similarly to [14]. Considering only downlink communications the total number of downlink control and data resources available per LTE frame is calculated based on the cell bandwidth. Next the model calculates the number of control and data resources required for the traffic profile defined using each possible modulation coding scheme (MCS). Unlike the number of data resources,

Table 1: Simulation Parameters

\begin{tabular}{|l|c|}
\hline \multicolumn{1}{|c|}{ Parameter } & Value \\
\hline Number of base stations (BS) & 30 \\
\hline Frequency (GHz) & 2 \\
\hline Bandwidth & 10 \\
\hline Downlink-uplink ratio & $1: 1$ \\
\hline Duplexing & TDD \\
\hline PDSCH Tx Power (dBm) & $15-30$ \\
\hline BS antenna gain $(\mathrm{dBi})$ & 16 \\
\hline BS height (m) & 40 \\
\hline User antenna gain (dBi) & 2 \\
\hline BS antenna pattern & $3-$ Sectored $[1]$ \\
\hline Cable loss (dB) & 3 \\
\hline Cyclic combing gain (dB) & 3 \\
\hline User noise figure (dB) & 8 \\
\hline Interference margin (dB) & 3 \\
\hline Receiver SINR requirements & {$[25.57 .912 .215 .5$} \\
(dB) & $17.5]^{\mathrm{a}}$ \\
\hline Control symbols per LTE frame & 4 \\
\hline
\end{tabular}

a: [1/2 QPSK 3/4 QPSK 16-QAM, 2/4 16-QAM, 2/3 64-QAM, 3/4 64-QAM] which varies with data MCS, the number of control resources required for a user will be constant because a single MCS is used for transmitting control resources in LTE. Thereafter, the total number of users who can achieve each MCS is calculated for the active subscriber list. From these numbers the distribution of MCSs between users in each sector is found. Finally, the theoretical maximum number of data users, $N_{D}$, and control users, $N_{C}$, may be found as follows:

$$
\begin{aligned}
& N_{D}=S_{\text {total }} \times\left(\frac{\sum_{i=1}^{n} P_{M C S_{i}} \times S_{M C S_{i}}}{n}\right)^{-1} \\
& N_{C}=\frac{C C E_{\text {total }}}{C C E_{\text {user }}}
\end{aligned}
$$

where $S_{\text {total }}$ is the total number of data resources available, $P_{M C S i}$ is the probability that a user uses $\mathrm{MCS}_{\mathrm{i}}, S_{M C S i}$ is the number of data resources required for $\mathrm{MCS}_{\mathrm{i}}$ to service the user's traffic demand, $n$ is the total number of MCS, $C C E_{\text {total }}$ is the total number of control resources, and $C C E_{\text {user }}$ is number of control elements required per user per packet transmission. From this, $C_{\text {users }}$ is calculated as:

$$
C_{\text {users }}=\min \left(N_{D}, N_{C}\right)
$$

This calculation of the number of connected users assumes the order in which users request connection is random. In addition the smart scheduler proposed in [14] is used to minimize the amount of control information required per user.

Based on the active subscriber list and the maximum number of possible users the connection success rate is calculated next. This is defined as the ratio of the maximum number of connected users to the total number of users in the active subscriber list.

The base station power consumption, $P_{B S}$, for a given transmit power is found using the model and parameters outlined in [10]. This model calculates a realistic power consumption value of a complete base station including factors such as air conditioning, microwave backhaul link, etc. Based on this and the coverage area the area power consumption is calculated as:

$\frac{P_{B S}}{A_{C}}$

The general parameters used for the simulation are provided in Table 1 . In addition the base station antenna radiation pattern and propagation models outlined in [1] were used. 


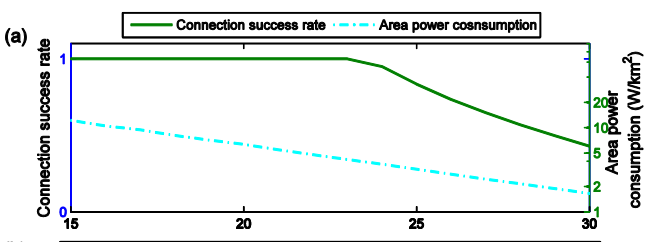

(b)

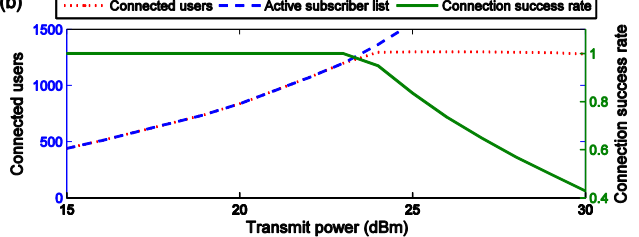

Figure 1: Transmit power for an 3-sectored base station with fixed bandwidth versus (a) connection success ratio and area power consumption and (b) connected users, active subscriber list, and connection success ratio

\section{b) Transmit power and area power consumption}

We first consider a scenario in which base station bandwidth is fixed at $10 \mathrm{MHz}$ and connection success rates less than 1 are not acceptable. The objective is to find the transmit power which corresponds to the optimum area power consumption, $P_{\text {Opt-APC}}$, subject to these constraints.

Figure 1a shows the relationship between transmit power, connection success rate, and area power consumption for a rural user density (10 users $/ \mathrm{km}^{2}$ ) and a VoIP traffic profile (40 bytes sent every $20 \mathrm{~ms}$ with $50 \mathrm{~ms}$ maximum latency) with a total base station bandwidth of $10 \mathrm{MHz}$. While the latest wireless standards, for example LTE and Mobile WiMAX, are being rolled out to provide high capacity services in mostly urban areas such standards will become increasingly important in providing coverage to rural environments. In addition it is foreseen that in the future operators will no longer use $2 \mathrm{G}$ standards such as GSM in their networks [6]. While this is likely to take time, eventually all mobile traffic including low data services such as voice will be accommodated by $3 \mathrm{G}$ and $4 \mathrm{G}$ standards, as has already happened in some countries, e.g. Japan. It is therefore relevant to examine the most cost effective and energy efficient way to support VoIP in LTE type cells.

It is obvious from Figure 1a that area power consumption decreases monotonically as transmit power is increased in this fixed bandwidth scenario. Therefore $P_{\text {Opt-APC }}$ is simply the largest transmit power which can achieve a connection success rate of 1 . Figure $1 \mathrm{~b}$ illustrates why the connection success rate eventually drops below 1 for increasing transmit power (all else remaining constant). As the transmit power in the cell is increased, the coverage area also increases. The increased coverage area results in a larger active subscriber list with a consequent increase in total traffic demand for the cell. As long as the base station can meet the traffic demands, the connection success rate is 1 . When the traffic demand exceeds the cell capacity for control or data resources it is no longer possible to connect all users seeking service and the connection success rate drops below 1 .

If the traffic demand in the cell increases, because of a higher density of users or an increase in the traffic demand per user, the value of $P_{O p t-A P C}$ will reduce in this constant bandwidth scenario. The reduced $P_{O p t-A P C}$ results in a smaller cell size and is consistent with the general philosophy that a higher density of smaller cells is required to provide increased traffic capacity [18]. Referring to Figure 1a it should be clear that a smaller cell will also result in an increased area power consumption and therefore a more costly, less energy efficient network.

An alternative approach to increasing capacity is to use more bandwidth. The effect of doing so and the effect of changing bandwidth on the area power consumption is examined next.

\section{c) Bandwidth and area power consumption}

For economic and other reasons the amount of spectrum available to operators is often limited. Nevertheless, there is usually some flexibility in the amount of bandwidth assigned to each base station. For this reason, this section will examine the optimum area power consumption when both base station bandwidth and transmit power are varied. Like the previous section, a connection success ratio less than 1 for the cell will be deemed unacceptable, and the same user density and traffic profile were used for all simulations.

When performing a downlink link budget analysis between a base station and mobile user, the bandwidth used in calculating the noise power and the receiver sensitivity is equal to the total bandwidth used by the base station or cell [19]. This is in contrast to the effective mobile user bandwidth which is used in an uplink link budget analysis. As the channel (downlink) bandwidth used by a base station increases the overall noise power will also increase, reducing the allowable propagation loss. Consequently the coverage area will reduce as the channel bandwidth increases, assuming transmit power remains constant. The reduced coverage area leads in turn to increased area power consumption and reduced energy efficiency. For this reason and for the general purpose of saving spectrum, unused or little used spectrum should be avoided. However in areas of high demand or to support a large coverage area it can be beneficial to use more bandwidth. 


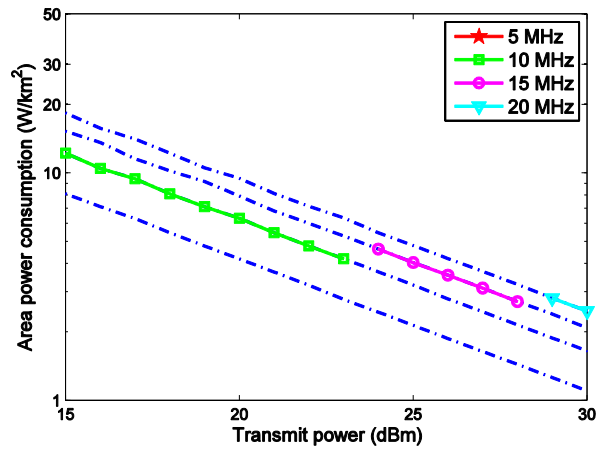

Figure 2: Area power consumption (dotted dashed lines) versus transmit power for bandwidths of 5 (lowermost line), 10, 15, and $20 \mathrm{MHz}$ (uppermost line). Markers indicate the optimum area power consumption subject to constraints at each transmit power

The relationship between bandwidth and area power consumption is shown in Figure 2. The area power consumption was calculated for each transmit power for bandwidths of 5, 10, 15 and 20 MHz. The dotted dashed lines in Figure 2 exhibit the pattern of monotonically decreasing area power consumption with increasing transmit power seen previously in Figure 1a. The connection success rate was calculated for every transmit power and bandwidth. At each transmit power the bandwidth which provided the lowest area power consumption while satisfying the connection success rate constraint was identified and these values are overlaid with coloured markers in Figure 2. The $5 \mathrm{Mhz}$ bandwidth did not satisfy the connection success rate constraint at any transmit power. At $15 \mathrm{dBm}$, a connection success rate of 1 can be achieved with all other bandwidths. Selecting the smallest bandwidth which satisfies the constraint $(10 \mathrm{MHz}$ in this case) minimizes the area power consumption. As the transmit power increases area power consumption for a given bandwidth reduces until the coverage area gets too large and the connection success rate constraint can no longer be satisfied, for example at $23 \mathrm{dBm}$ for $10 \mathrm{MHz}$ bandwidth. At this point, choosing the next largest bandwidth satisfies the constraint again and the transmit power (and coverage area) of the cell can be increased once more.

As a result of switching bandwidths, the area power consumption no longer decreases monotonically as transmit power increases. Nevertheless, the transmit power corresponding to the overall optimum area power consumption for the cell, $P_{\text {Opt-APC}}$, is still the maximum transmit power which provides full user coverage: $30 \mathrm{dBm}$ in this case with a corresponding bandwidth of 20 $\mathrm{MHz}$. The area power consumption at this point is approximately $2.47 \mathrm{~W} / \mathrm{km}^{2}$ representing a reduction of $54 \%$ compared to the fixed $10 \mathrm{MHz}$ case shown in Figure 1a. This result shows that larger cells with larger bandwidth allocations can be used to improve the energy efficiency of the network. Although the spectrum to permit increased bandwidth may not always be available this finding highlights an important trade-off that exists in the design of a base station between the achievable area power consumption and the amount of bandwidth used.

\section{A MODIFIED CONNECTION SCHEME}

In [16] a modified connection scheme was presented as a mechanism for increasing the spectral efficiency of a base station without requiring increased bandwidth or additional infrastructure. An increase in spectral efficiency leads to an increased traffic capacity in the cell. The question is, can this increased capacity also be used to improve the area consumption of the cell?

\section{a) Modified connection scheme description}

The edge SINR is the minimum SINR threshold which a user must reach in order to be connected to the base station and is therefore the SINR which defines the edge of the coverage area. The modified connection scheme permits the maximum spectral efficiency achievable within a cell to be increased by increasing the edge SINR. By increasing this value the fraction of users using the lowest order modulation and coding scheme (MCS) is reduced. This results in more data resources being available to higher order MCS users. Since resources allocated to a higher order MCS can carry more data than those allocated to a lower order MCS, the cell capacity is increased. The increased capacity can be used to connect more users with a given traffic profile, or to handle an increased traffic demand per user.

Figure 3 shows the effect of increasing the edge SINR. The stacked area plot represents the relative contributions of each MCS to the total spectral efficiency as indicated by the legend. As the edge SINR threshold is increased, the relative contribution of lowest order MCS decreases while the other MCS contributions increase. This leads to increased spectral efficiency, assuming the same underlying conditions as the previous sections, namely a uniformly distributed population of users and users connecting in random order. As the edge SINR continues to be increased, eventually only the highest order MCS remains at which point the spectral efficiency can spectral efficiency which can be achieved for the frame structure and bandwidth used. 


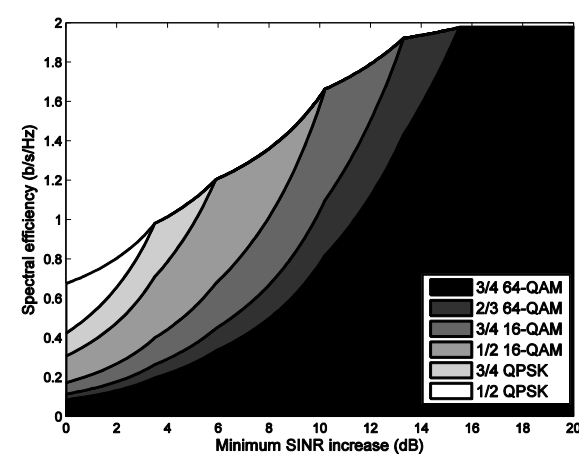

Figure 3: The relationship between spectral efficiency and increased edge SINR. Each area shows the relative contribution of that MCS to the total

\section{b) Edge SINR and area power consumption}

If user density and traffic profile remain constant and if the cell capacity is not control resource limited, then the modified connection scheme permits an increased number of connected users which in turn permits a larger coverage area than would otherwise be possible. To achieve the increased coverage area and ensure the connection success rate remains at 1 , two increases in transmit power are required. First the transmit power must be increased to achieve the increased coverage area. Second, the transmit power must be increased by the amount of the edge SINR increase. This two increases together ensure that the SINR at the edge of the intended coverage area is the modified edge SINR required for connection. This can be summed up with the following equation:

$$
\Delta P_{\text {total }}=\Delta P_{\text {area }}+\Delta P_{S E-\text { Increase }}
$$

where $\Delta P_{\text {total }}$ is the total transmit power increase required, $\Delta P_{\text {area }}$ is the transmit power increase required to provide a larger coverage area, and $\Delta P_{S E-I n c r e a s e}$, is the transmit power increase required to increase spectral efficiency. Ultimately the achievable gain in coverage area and cell capacity is limited by the maximum transmit power that can be used in the system.

When using the modified connection scheme to increase the achievable coverage area, the area power consumption can be reduced compared the unmodified scheme. The reason for this is that the rate at which the coverage area increases is greater than the rate at which the base station power consumption increases, despite the additional transmit power required to match the increase in the edge SINR. This is demonstrated in the results presented in Figure 4 and Table 2.

In Figure 4 the optimum area power consumption was calculated for various increases

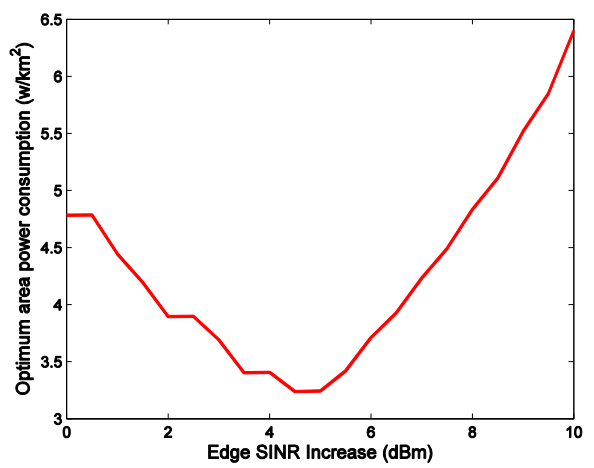

Figure 4: Optimum area power consumption calculated for different levels of edge SINR increase

in the edge SINR using the same methodology and underlying conditions as in Figure 1a. Without any edge SINR increase the optimum area power consumption was calculated to be $4.78 \mathrm{~W} / \mathrm{km}^{2}$ with a transmit power of $22 \mathrm{dBm}$ (corresponding to the optimum in Figure 1a). With the modified connection scheme this was reduced to 3.24 $\mathrm{W} / \mathrm{km}^{2}$ with a optimum transmit power of 29.5 $\mathrm{dBm}$ and an increase to the edge SINR of 4.5 $\mathrm{dBm}$. This represents a $32 \%$ decrease in area power consumption and therefore a significant reduction in the energy cost of the base station design. It should also be noted that both the coverage area and connected users have increased significantly as can be seen in Table 2 .

The increased cell capacity using the modified scheme (indicated by the increase in connected users) is approximately $50 \%$ in this case. In practice the achievable gain in capacity is limited by the number of control resources available per LTE frame. In LTE the number of control resources available per frame is fixed. Eventually, for a large number of users, all available control resources will be allocated and no more users can be connected despite the possible availability of unused data resources. The

Table 2: Simulation Results

\begin{tabular}{|l|c|c|}
\hline \multicolumn{1}{|c|}{ Parameter } & $\begin{array}{c}\text { Without } \\
\text { edge SINR } \\
\text { increase }\end{array}$ & $\begin{array}{c}\text { With edge } \\
\text { SINR } \\
\text { increase }\end{array}$ \\
\hline $\begin{array}{l}\text { Optimum tx } \\
\text { power (dBm) }\end{array}$ & 22 & 29.5 \\
\hline $\begin{array}{l}\text { Bandwidth } \\
\text { (MHz) }\end{array}$ & 10 & 10 \\
\hline $\begin{array}{l}\text { Area power } \\
\text { consumption } \\
\text { (W/km }{ }^{2} \text { ) }\end{array}$ & 4.78 & 3.24 \\
\hline Coverage area & 126.75 & 189 \\
\hline $\begin{array}{l}\text { Edge SINR } \\
\text { increase (dBm) }\end{array}$ & 0 & 4.5 \\
\hline $\begin{array}{l}\text { Connected } \\
\text { users }\end{array}$ & 1195 & 1849 \\
\hline
\end{tabular}


VoIP traffic profile considered in this work has a relatively high control overhead, and higher data rate traffic profiles with relatively less control overhead could be expected to exhibit better gains.

There are two features of Figure 4 worth highlighting. First, for edge SINR increases above $4.5 \mathrm{~dB}$ the optimum area power consumption increases again. This occurs when $\Delta P_{\text {area }}$ cannot achieve its desired value either because there are insufficient control resources to support the larger cell size or because the transmit power is capped at its maximum value. In either case, the result is that the increased edge SINR results in wasted power that increases the area power consumption. Second, the steps in the area power consumption are due to the resolution at which the transmit power is increased in the model. This resolution impacts not only the resolution at which the coverage area and area power consumption can change, but also the resolution at which the edge SINR can increase and therefore the achievable reduction in area power consumption. For the purposes of illustration in this paper the transmit power is increased in steps of $0.5 \mathrm{dBm}$ however more realistic values are $1 \mathrm{dBm}$ [20].

It is important to note that even with large step increases in transmit power an accurate estimation of SINR is required by mobile user in the downlink and base station in the uplink. In LTE the channel quality indicator (CQI) parameter can be used to help estimate the receiver SINR and the appropriate MCS which should be used. The proposed modified admission scheme would require a greater resolution of channel quality estimation than is currently possible using the CQI method of feedback however as has been shown the benefits of the modified admission scheme in reducing the potential network energy cost is significant.

In summary the results show that the area power consumption of a base station can be reduced significantly by using the modified connection scheme. When such a solution is extended to the overall network it will result in a direct saving in total energy costs. Based on these findings it appears that the edge SINR is an important parameter to include in the design and optimization of base stations, particularly in green field deployments such as new LTE networks. In addition energy savings may also be possible through the reconfiguration of this parameter in existing networks. However the possibility of any savings will very much depend on the traffic demands of the network and flexibility of the network with regards to changes. The optimization of the edge SINR parameter within existing networks is an area of future work.

\section{CONCLUSION}

In this paper area power consumption was optimized subject to constraints for a single 3sectored LTE type base station in a multi base station network. Section II b showed that when bandwidth is constant, the transmit power which optimized area power consumption subject to maintaining a connection success rate of 1 was simply the maximum transmit power which permits this connection success rate. Consequently, the optimum area power consumption is achieved by designing for the largest coverage area which satisfies the constraints. Section II c showed that when bandwidth is increased, a larger coverage area can be supported and hence the area power consumption can potentially be reduced further. Finally, section III showed that a modified connection scheme can be used to improve cell capacity for a given bandwidth and that this approach can also be used to increase coverage area and decrease area power consumption. Though not specifically discussed, the schemes can be combined to yield the lowest possible area power consumption provided the base station does not become control or data resource limited. It is worth emphasizing the point that although the optimizations were carried out for a single base station in a simplified multi base station network, the trend of the findings will extend to a more realistic network and consideration of these findings can permit significant reductions in the total power consumption of a mobile network.

\section{ACKNOWLEDGEMENTS}

The authors wish to thank Jean-Christophe Schiel and François Montaigne for their assistance and support. Also the authors extend thanks to the sponsors EADS and IRCSET for the $\mathrm{PhD}$ program.

\section{REFERENCES}

[3] Cisco, "Cisco visual network index: Global mobile data traffic forecast update, 2012-2017," White paper, 2013.

[4] A. U. Mikko, "Global Vision for the Future Wireless World from the WWRF," Vehicular Technology Magazine, IEEE, vol. 1, pp. 4-8, 2006. 
[5] M. Dohler, et al., "Is the PHY layer dead?," Communications Magazine, IEEE, vol. 49, pp. 159-165, 2011.

[6] W. Webb, Wireless Communications: The Future: Wiley, 2007.

[7] Vodafone, "The journey to sustainability: Reporting on the Green Agenda, Vodafone Ireland's key sustainability programme," 2010.

[8] F. Richter, et al., "Energy Efficiency Aspects of Base Station Deployment Strategies for Cellular Networks," in Vehicular Technology Conference Fall (VTC 2009-Fall), 2009 IEEE 70th, 2009, pp. 1-5.

[9] EARTH, "project deliverable D2.4 v1.0, Most suitable efficiency metrics and utility functions."

[10] M. Deruyck, et al., "Modelling and optimization of power consumption in wireless access networks," Computer Communications, vol. 34, pp. 2036-2046, 2011.

[11] X. Jie and Q. Ling, "Area Power Consumption in a Single Cell Assisted by Relays," in Green Computing and Communications (GreenCom), 2010 IEEE/ACM Int'l Conference on \& Int'l Conference on Cyber, Physical and Social Computing (CPSCom), 2010, pp. 460-465.

[12] F. Richter, et al., "Traffic Demand and Energy Efficiency in Heterogeneous Cellular Mobile Radio Networks," in Vehicular Technology Conference (VTC 2010-Spring), 2010 IEEE 71st, 2010, pp. 1-6.

[13] O. Arnold, et al., "Power consumption modeling of different base station types in heterogeneous cellular networks," in Future Network and Mobile Summit, 2010, 2010, pp. 1-8.

[14] S.-I. Chakchai, et al., "Capacity Evaluation for IEEE 802.16e Mobile WiMAX," ed: Hindawi Publishing Corporation, 2010.

[15] N. A. El-fishawy, et al., "VoIP capacity estimation in mobile WiMAX networks," in Radio Science Conference (NRSC), 2011 28th National, 2011, pp. 1-10.

[16] T. Keenan and R. Villing, "Maximising spectral efficiency in LTE cells," Irish Signals and System Conference, 2012.

[17] T. Keenan and R. Villing, "Evaluating wireless cell capacity," RIA Colloquium on Communications and Radio Science into the 21st Century, 2012.

[18] B. Badic, et al., "Energy Efficient Radio Access Architectures for Green Radio:
Large versus Small Cell Size Deployment," in Vehicular Technology Conference Fall (VTC 2009-Fall), 2009 IEEE 70th, 2009, pp. 1-5.

[19] H. Holma and A. Toskala, "LTE for UMTS - OFDMA and SC-FDMA Based Radio Access," ed: Wiley, 2009, pp. 225226.

[20] A. Molisch, Wireless Communications, Second ed.: Wiley, 2011. 